\title{
Shell model in the complex energy plane and two-particle resonances
}

\author{
R. Id Betan, ${ }^{1,2}$ R. J. Liotta, ${ }^{1}$ N. Sandulescu, ${ }^{1,3}$ and T. Vertse ${ }^{1,4}$ \\ ${ }^{1}$ Royal Institute of Technology, Alba Nova, SE-10691 Stockholm, Sweden \\ ${ }^{2}$ Departamento de Fisica, FCEIA, UNR, Avenida Pellegrini 250, 2000 Rosario, Argentina \\ ${ }^{3}$ Institute of Physics and Nuclear Engineering, P.O.Box MG-6, Bucharest-Magurele, Romania \\ ${ }^{4}$ Institute of Nuclear Research of the Hungarian Academy of Sciences, H-4001 Debrecen, P.O. Box. 51, Hungary
}

(Received 14 August 2002; published 31 January 2003)

\begin{abstract}
An implementation of the shell-model to the complex energy plane is presented. The representation used in the method consists of bound single-particle states, Gamow resonances and scattering waves on the complex energy plane. Two-particle resonances are evaluated, and their structure in terms of the single-particle degrees of freedom is analyzed. It is found that two-particle resonances are mainly built upon bound states and Gamow resonances, but the contribution of the scattering states is also important.
\end{abstract}

DOI: 10.1103/PhysRevC.67.014322

PACS number(s): 25.70.Ef, 23.50.+z, 25.60.-t, 21.60.Cs

\section{INTRODUCTION}

The study of processes that takes place in the continuum part of nuclear spectra is a difficult undertaking. This can be seen by the large number of methods that have been proposed to describe the continuum. In addition to the Feshbach theory [1] and its many versions, there are many methods where the continuum is described by a finite set of positive energy states. These states are usually obtained by expanding the solutions of the nuclear Hamiltonian in a harmonicoscillator basis or by solving the eigenvalue problem with box boundary conditions. There have been also many variations of these procedures, e.g., by using a transformed harmonic-oscillator basis [2] or harmonic oscillators with different frequencies [3]. The common feature of these representations is that they are constrained to real energy solutions, as required by quantum mechanics. However, the widths corresponding to decay processes were already evaluated by means of outgoing waves (and therefore complex energies) by Gamow in his seminal paper on barrier penetration [4]. The Gamow states provide a natural definition of resonant states [5].

Berggren [6], and shortly afterward Romo [7], showed that the bound and Gamow states, together with a set of complex scattering states, form a representation which spans the space of complex energies. In this representation the scalar product (the metric) is non-Hermitian, and the norm of the Gamow states is defined by using a method introduced by Zel'dovich [8]. Later on, techniques based on the complex rotation of the radial distance were also used to regularize the Gamow states and the matrix elements involving them [9].

Microscopical calculations based on a single-particle representation consisting of bound states, Gamow resonances, and complex energy scattering states (Berggren representation) were proposed some years ago $[10,11]$. This representation was recently expanded to study two-particle resonances $[12,13]$. It may be surprising to see that it took such a long time for us (after Ref. [11]) to arrive at the two-particle representation. One of the reasons for this delay is that only recently have we managed to find a method which allows one to isolate the physical two-particle resonant states from the continuous background, as schematically outlined in Ref. [12]. The problem is that only a small fraction of the calculated states are physically relevant, since most of them (if not all) are either wide resonances or part of the nonresonant continuum. It is therefore important to be able to isolate the physically meaningful states from the rest of the spectrum. In this paper we will show how to achieve this. We will also show how two-particle resonances are built from the singleparticle degrees of freedom determining the dynamics of the system. This is important since it is not clear how a twoparticle resonance is formed. For instance, one may wonder to what extent such a state is built upon particles moving in resonant states as well as in nonresonant continuum states. Intuitively one would say that for the physically relevant two-particle resonances one of the two particles is in a narrow Gamow state while the other moves in a bound state. Wide resonances and the nonresonant continuum would play only a minor role, as assumed in Refs. [14-16]. We will show that this intuitive assumption is not always supported by proper calculations.

Actually the question of the importance of the nonresonant continuum in the calculations was usually skipped in relation to processes taking place in the continuum part of the spectrum, particularly regarding radioactive decay, where measurable lifetimes correspond to very narrow resonances. Therefore, a calculation of the corresponding decay widths can be performed by using bound representations, and the continuum itself can be totally ignored [17]. This approximation, which was followed in nearly all available calculations of cluster decay (including alpha particles and recent calculations of proton decay [18-21]) was very successful in explaining experimental data [22]. However, with the development of experimental facilities one could measure partial decay widths of neutrons from giant resonances, and the continuum had to be included explicitly in the formalism [23-25].

Even more important, the experimental discovery of halo nuclei triggered a very fruitful theoretical activity which showed that halos cannot be understood without taking into account wide resonances and other elements belonging to the continuum $[26,27]$. All these elements are automatically included in the representation presented in this paper. The 


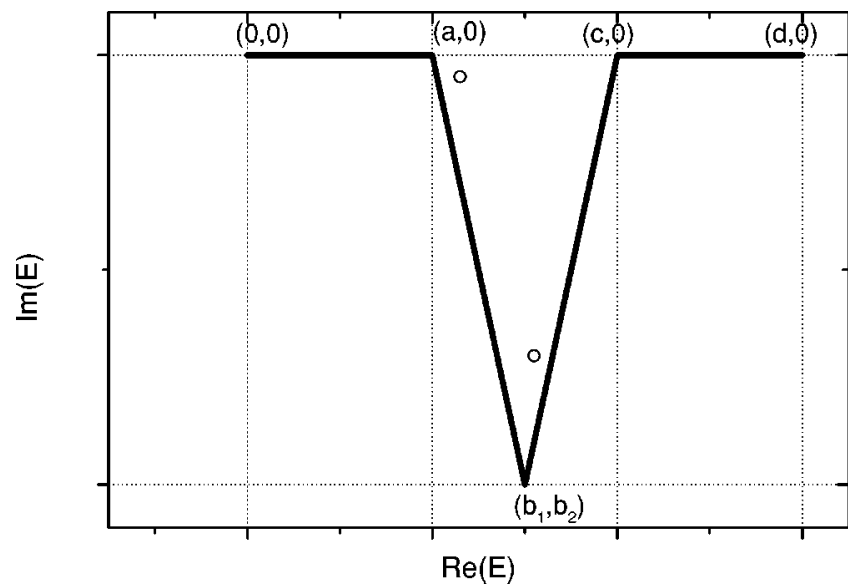

FIG. 1. One-particle complex energy plane. It shows the contour $\mathrm{L}^{+}$corresponding to the energy of the scattering waves (full line) and the Gamow resonances enclosed by the contour (open circles) defining the one-particle Berggren representation. The bound states, which enter in the representation independently of the contour, are not shown.

method, which is a generalization of the shell model to the complex energy plane, was briefly given in Ref. [12]. We will present it in detail here, clarifying what a two-particle resonance is. We will also provide an insight into the influence of the continuum upon the formation of two-particle resonances.

The formalism is shown in Sec. II. In Sec. III we give the applications and a summary, and conclusions are given in Sec. IV.

\section{FORMALISM}

The Berggren (one-particle) representation to be used in this work was described before in a number of situations, e.g., in Refs. $[6,10,11,25]$. Here we will give only a brief summary of the formalism.

The regular solutions of the Schrödinger equation, with outgoing boundary conditions corresponding to a particle moving in a central potential, provide the single-particle bound states and the so-called Gamow resonances. The study of this case led $[6,7]$ to the introduction of expansions for the Green and $\delta$ functions in terms of the poles of the Green function plus an integral along a continuum path in the complex energy plane, i.e.,

$$
\delta\left(r-r^{\prime}\right)=\sum_{n} w_{n}(r) w_{n}\left(r^{\prime}\right)+\int_{L^{+}} d E u(r, E) u\left(r^{\prime}, E\right),
$$

where we choose the integration path $L^{+}$to lie in the fourth quadrant of the complex wave number $(k)$ plane, ${ }^{1}$ as seen in Fig. 1. The summation runs over all bound states and poles of the Green function (Gamow resonances) enclosed by the

\footnotetext{
${ }^{1}$ This corresponds to the lower half of second, nonphysical Rieman $E$ sheet.
}

real $E$ axis and the contour $L^{+}$. One can choose quite general forms for the contour, as can be seen in Ref. [10], but it has to go through the origin and finish at infinite on the real energy axis. However, as in any shell-model calculation, one cuts the energies at a certain maximum value, which in Fig. 1 corresponds to the point $D \equiv(d, 0)$. The other points defining the contour in that figure are, besides the origin, $A$ $\equiv(a, 0), B \equiv\left(b_{1}, b_{2}\right)$ and $C \equiv(c, 0)$.

In Eq. (1) the scattering functions on the contour are denoted by $u(r, E)$, while the wave functions of the bound single-particle states and the Gamow resonances are denoted by $w_{n}(r)$. These states are indicated by open circles in Fig. 1.

An important feature in Eq. (1) is that the scalar product is defined as the integral of the wave function times itself, and not its complex conjugate. This is in agreement with the Hilbert metric on the real energy since, for bound states or for scattering states on the real $E$ axis, one can choose the phases such that the wave functions are real quantities. The prolongation of the integrand to the complex energy plane allows one to use the same form for the scalar product everywhere. This metric (Berggren metric) produces complex probabilities, as has been discussed in detail in, e.g., Refs. $[17,23]$. Here it is worthwhile to point out that for narrow resonances such probabilities become virtually real quantities.

The integral in Eq. (1) can be discretized such that

$$
\int_{L^{+}} d E u(r, E) u\left(r^{\prime}, E\right)=\sum_{p} h_{p} u\left(r, E_{p}\right) u\left(r^{\prime}, E_{p}\right)
$$

where $E_{p}$ and $h_{p}$ are defined by the procedure one uses to perform the integration. In the Gaussian method $E_{p}$ are Gaussian points and $h_{p}$ the corresponding weights. Therefore, the orthonormal (in the Berggren metric) basis vectors $\left|\varphi_{j}\right\rangle$ are given by the set of bound and Gamow states, i.e. $\left\langle r \mid \varphi_{n}\right\rangle=\left\{w_{n}\left(r, E_{n}\right)\right\}$, and the discretized scattering states, i.e., $\left\langle r \mid \varphi_{p}\right\rangle=\left\{\sqrt{h_{p}} u\left(r, E_{p}\right)\right\}$. This defines the Berggren representation.

By using the Berggren representation one readily gets the two-particle shell-model equations, i.e.,

$$
\left(\omega_{\alpha}-\epsilon_{i}-\epsilon_{j}\right) X(i j ; \alpha)=\sum_{k \leqslant l}\langle\widetilde{k} l ; \alpha|V| i j ; \alpha\rangle X(k l ; \alpha),
$$

where $\alpha$ labels two-particle states, and $i, j, k$, and $l$ label single-particle states. The tilde denotes mirror states [6] and the rest of the notation is standard.

As usual, the two-particle wave function is

$$
|\alpha\rangle=\sum_{i \leqslant j} X(i j ; \alpha)\left(c_{i}^{+} c_{j}^{+}\right)_{\alpha}|0\rangle,
$$

where 


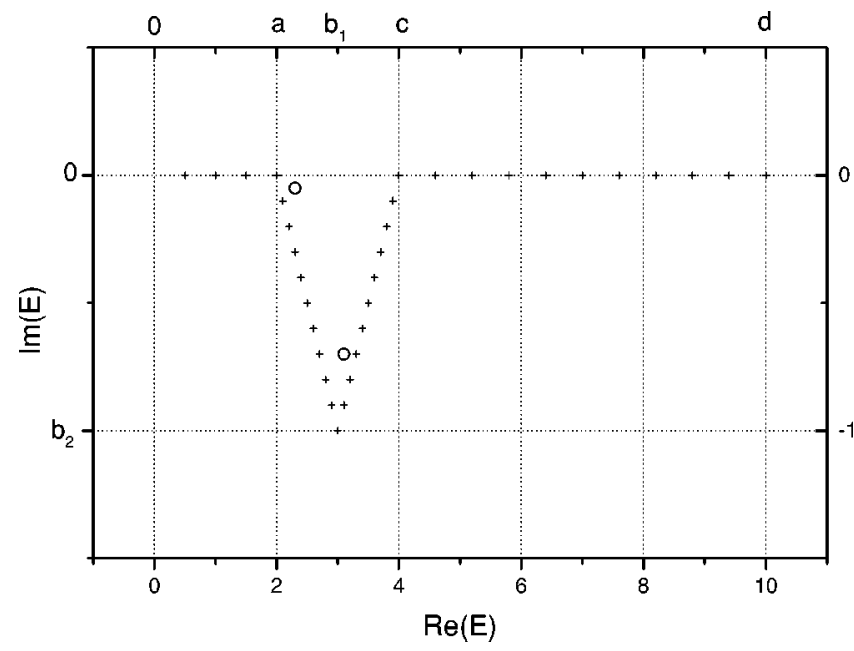

FIG. 2. Discretized contour and Gamow resonances defining the one-particle Berggren representation. The open circles indicate the energies of the Gamow resonances while the crosses are the energies of the scattering states.

$$
X(i j ; \alpha)=\left\langle\alpha\left|\left(c_{i}^{+} c_{j}^{+}\right)_{\alpha}\right| 0\right\rangle /\left(1+\delta_{i j}\right)^{1 / 2} .
$$

The discretization of the contour in Fig. 1 produces a series of points in the one-particle energy plane, as shown in Fig. 2. Each point in this figure represents a state of our one-particle Berggren representation. Therefore, the energy of the twoparticle basis vector $\left(c_{i}^{+} c_{j}^{+}\right)_{\alpha}|0\rangle$, i.e., $\epsilon_{i}+\epsilon_{j}$, is the sum of the point $i$ in Fig. 2 with the point $j$. Allowing the indices $i$ and $j$ to run over all the one-particle basis states, ordered such that $i \leqslant j$, one obtains the energies of the two-particle basis states (zeroth-order energy) in the corresponding (twoparticle) complex energy plane as shown in Fig. 3. One sees in this figure that the whole complex energy plane of interest is covered by zeroth-order solutions and, therefore, it would be difficult in this plane to find the physical two-particle

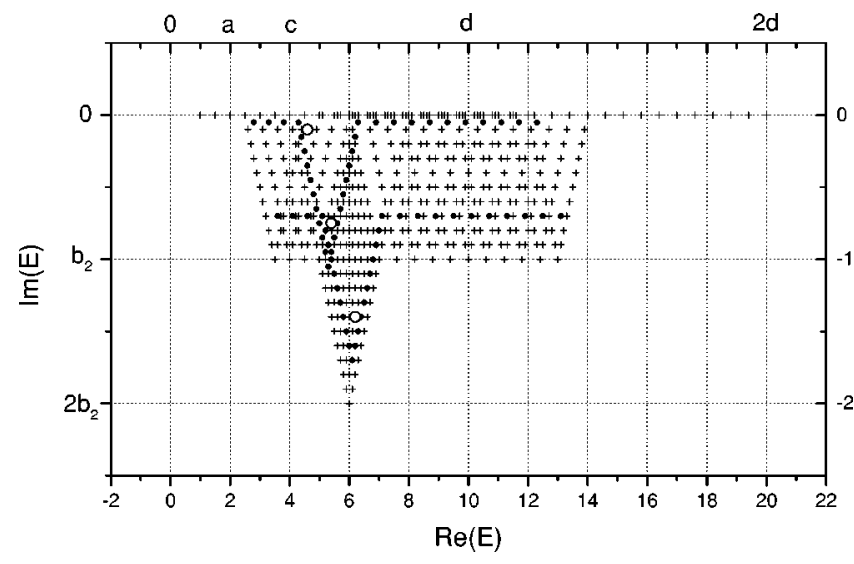

FIG. 3. Zeroth-order two-particle energy points obtained from the one-particle states in Fig. 2. These points define the two-particle Berggren representation. The open circles correspond to the cases in which both particles occupy Gamow states. The dots are the energies in which one particle is in a Gamow state while the other is in a scattering state. The crosses are the energies corresponding to the cases in which both particles are in scattering states.

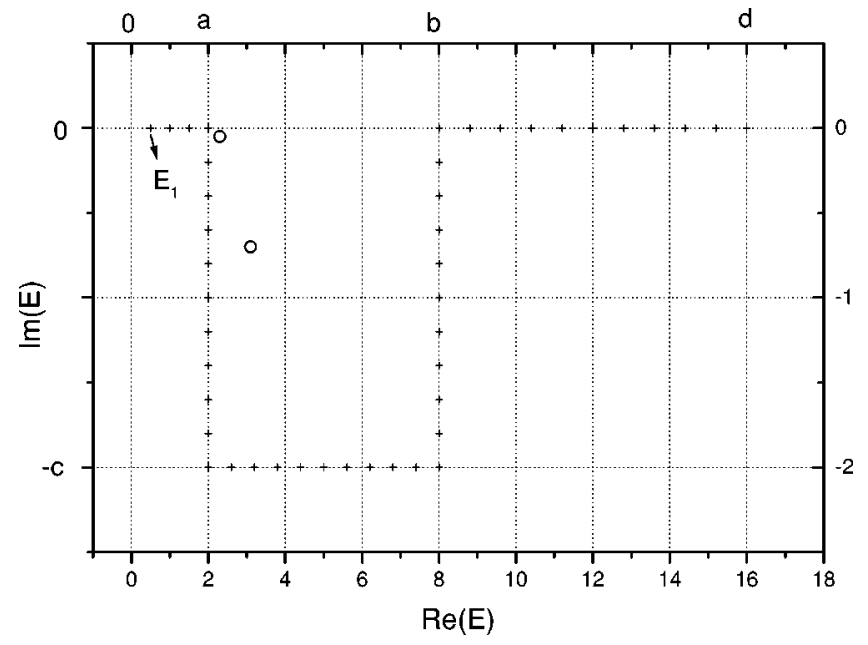

FIG. 4. One-particle discretized contour which produces the two-particle energy region free of zeroth order states shown in Fig. 5. The open circles indicate the energies of the Gamow resonances while the crosses are the energies of the scattering states. Note that the point $(0,0)$ does not belong to the representation. The lowest energy corresponding to scattering basis states lies at $\left(E_{1}, 0\right)$.

states. This problem becomes more acute as the number of elements in the basis increases, that is as the number of points in Fig. 2 becomes larger, because the two-particle physical states would then be embeded in a dense number of states belonging to the continuum.

A way to avoid this problem is to choose a one-particle contour which leaves a physically relevant two-particle complex energy region free of zeroth order states. There are many possible contours that satisfy this condition. In Fig. 4 we show one such contour and the corresponding Gamow resonances enclosed by it. Since the bound states have to be considered in any case, irrespective of the contour, we do not include them in the discussion here.

The contour has a rectangular form defined by the points $P_{0} \equiv(0,0), P_{1} \equiv(a, 0), P_{2} \equiv(a,-c), P_{3} \equiv(b,-c), P_{4} \equiv(b, 0)$, and $P_{5} \equiv(d, 0)$. As mentioned above, the number $d$ should be infinite. However, if one chooses $d$ large enough its value does not influence significantly the calculated quantities of interest. In the cases to be studied in Sec. III we will choose $d$ fulfilling such a condition. It is also worthwhile to mention that, due to the Gaussian integration method, the point $(0,0)$ will not belong to the Berggren representation. The lowest energy on the contour corresponds to the first Gaussian point, i.e., $\left(E_{1}, 0\right)$.

By summing in an orderly manner the points of this figure with themselves, one obtains the two-particle states shown in Fig. 5. One can see that if $2 a<b$ then there is a region in the two-particle complex energy plane which is practically free of any uncorrelated solution. Choosing the real energies $a$ and $b$ in Fig. 4 conveniently, one can study two-particle resonances lying in any reasonable energy region. We will call this the "allowed" energy region. In Fig. 5 all possible twoparticle energy points have been drawn. The allowed region occupies a rather small portion of the figure, and therefore it appears somehow diffuse among all the points. For clarity of 


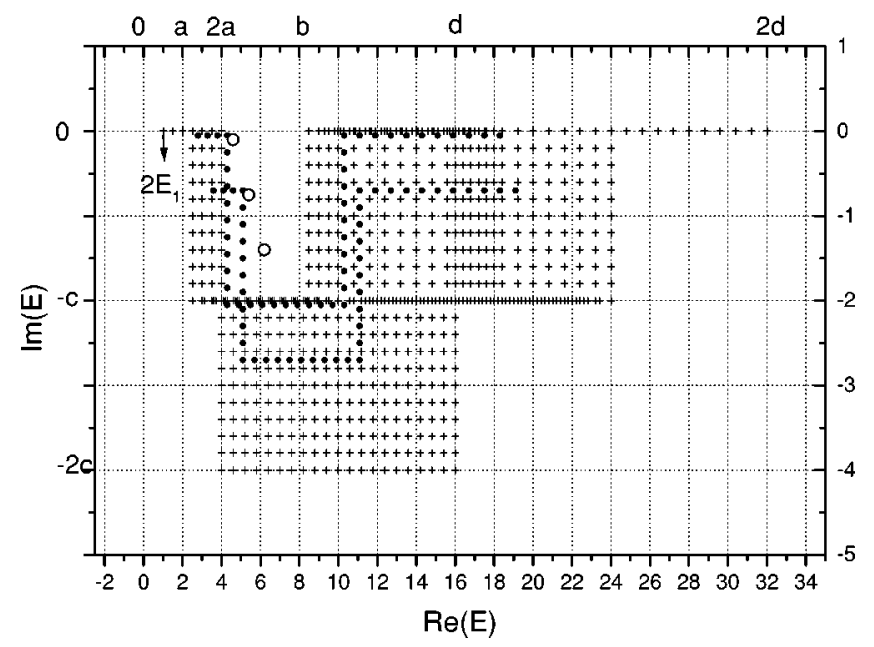

FIG. 5. Energies of the uncorrelated two-particle states obtained from the one-particle energies of Fig. 4. The open circles correspond to the cases in which both particles occupy Gamow states. The dots are the energies in which one particle is in a Gamow state while the other is in a scattering state. The crosses are the energies corresponding to the cases in which both particles are in scattering states. The allowed region is the one with real energy between $2 \mathrm{a}$ and $b$ and with imaginary energy larger than $-c$. The basis vector with lowest energy lies at $2 E_{1}$.

presentation in Fig. 6 we show the allowed region and its neighborhood only.

The allowed region can be determined by fulfilling some physically meaningful requirements. That is, the correlated states of interest are those which live a long time before decaying. One would thus be able to observe them directly or through effects that they induce, for instance through the formation of halos. As we will show below, such states are built mainly by Gamow resonances. Therefore, the contour in Fig. 4 should be chosen in such way as to enclose those resonances. Moreover, the values of $a, b$, and $c$ should produce an allowed region where the calculated two-particle states may lie. Since the two-body interaction is attractive

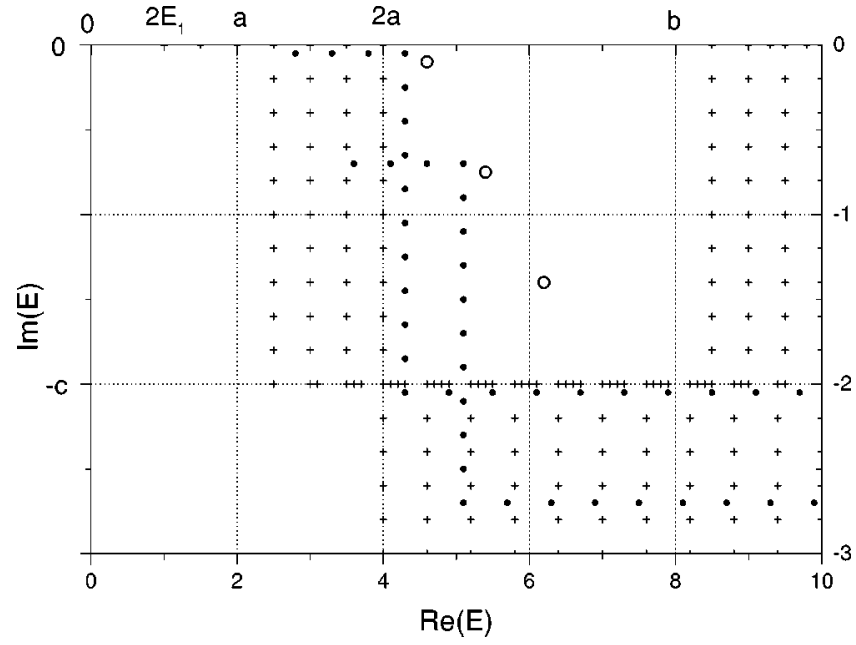

FIG. 6. As in Fig. 5, but enlarged such that only the allowed region and its neighborhood are included. one expects that the correlated low-energy resonances would lie below their zeroth-order positions in Fig. 6. Therefore, the value of $2 a$ should be as small as possible while $b$ should be large, which are just the conditions needed to obtain the allowed region.

Once the Berggren single-particle representation has been chosen, the two-particle representation is built as in the standard shell model, i.e., as the tensorial product of the oneparticle representation with itself. This defines the twoparticle space. In this space there are, in zeroth-order, twoparticle configurations containing Gamow resonances only, which in Fig. 6 are indicated by open circles. Besides these, one sees that inside the allowed region there are also configurations, indicated by dots, corresponding to one particle moving in a Gamow resonance and the other in continuum states. These configurations can be seen more clearly in Fig. 5 , where the dots form two recognizable rectangles. Each rectangle corresponds to the geometrical sum of a Gamow resonance, represented by an open circle in Fig. 4, and the scattering functions on the contour, represented by the crosses in that figure. In addition to the two-particle states involving Gamow resonances there are also configurations, denoted by crosses in Fig. 5, in which both particles are in single-particle scattering states.

One expects that physically relevant resonances are mostly determined by two-particle configurations in which both particles are in either bound or Gamow states. Since these states are the ones expected from the standard shell model, we will call them "resonant shell model" states.

In the applications shown in this paper the central field determining the single-particle basis will be of a WoodsSaxon type with a spin-orbit term as in Ref. [28]. The corresponding bound and Gamow functions as well as the scattering states will be evaluated by using the computer codes described in Refs. [28,29].

As a two-body interaction we will use a separable twobody force given by the derivative of the Woods-Saxon potential. This effective interaction has shown to be satisfactory to describe processes in the continuum, e.g., neutron decay from giant resonances [23]. However, our purpose here is not to explain physical processes in the continuum in detail, but rather to understand the role played by the various ingredients entering into the calculations. The great feature of the separable force is that it does not require a diagonalization of matrices. This is important in our case since the dimension of the two-particle basis may be very large, and we want to evaluate all states in order to examine the distribution of the energies of the resonant shell model and scattering states discussed above.

Our effective interaction matrix element is then [30]

$$
\langle\widetilde{k} l ; \alpha|V| i j ; \alpha\rangle=-G_{\alpha} f_{\alpha}(k l) f_{\alpha}(i j),
$$

where

$$
\begin{aligned}
f_{\alpha}(p q)= & \frac{1+(-1)^{l_{p}+l_{q}-\lambda_{\alpha}}}{2 \sqrt{4 \pi}} \\
& \times(-1)^{j_{q}+1 / 2} \hat{\lambda}_{\alpha} \hat{j}_{p} \hat{j}_{q}\left\langle j_{p} 1 / 2 \quad \lambda_{\alpha} 0 \mid j_{q}-1 / 2\right\rangle \\
& \times \int_{0}^{\infty} d r \varphi_{p}(r)[r \partial U(r) / \partial r] \varphi_{q}(r),
\end{aligned}
$$


and $U(r)$ is the volume part of the Woods-Saxon potential defining the central field. The rest of the notation is standard. From Eqs. (3) and (6a) one obtains the dispersion relation

$$
-\frac{1}{G_{\alpha}}=\sum_{i \leqslant j} \frac{f_{\alpha}^{2}(i j)}{\omega_{\alpha}-\epsilon_{i}-\epsilon_{j}}
$$

which allows one to evaluate the correlated energies $\omega_{\alpha}$.

Note that in Eq. (6b) the angular part of the matrix element was evaluated according to the Hilbert metric, while in the radial part the Berggren metric is used. Note also that in Eq. (7) the square of the matrix element appears, and not the square of its absolute value.

In the case of a separable force the amplitude of the wave function can be written as

$$
X(i j ; \alpha)=N_{\alpha} \frac{f_{\alpha}(i j)}{\omega_{\alpha}-\epsilon_{i}-\epsilon_{j}},
$$

where $N_{\alpha}$ can be determined by the normalization condition, i.e.,

$$
N_{\alpha}^{-2}=\sum_{i \leqslant j}\left(\frac{f_{\alpha}(i j)}{\omega_{\alpha}-\epsilon_{i}-\epsilon_{j}}\right)^{2}
$$

We will determine the strength of the separable force, i.e., $G_{\alpha}$, by using the usual procedure of fitting the energy of a two-particle state $\alpha$. However, in the drip line nuclei which we will analyze there are not yet any experimental data, and, therefore, we will assume that such a state lies at a certain reasonable energy. We will also vary the value of $G_{\alpha}$ thus obtained within a reasonable range in order to study the influence of the two-body interaction upon the calculated states.

\section{APPLICATIONS}

We will apply the formalism discussed above for two cases, one corresponding to a nucleus close to the neutron drip line and the other close to the proton drip line. In both nuclei we will analyze two-particle states with an angular momentum $\lambda=0$. All partial waves with $l \leqslant 10$ will be included to evaluate the scattering states in the Berggren representation.

\section{A. Two-neutron resonances}

In this section we will present calculations for twoneutron states in the double closed-shell nucleus ${ }^{78} \mathrm{Ni}$. The Woods-Saxon parameters are indicated in Table I, and the corresponding single-particle energies are in Table II. These single-particle states are quite similar to the ones given by a Skyrme HF calculations [31]. As seen in Table II, the shell $N=50$ is well defined, since there is a gap of about $3.6 \mathrm{MeV}$ between the lowest particle state, which here is $1 d_{5 / 2}$, and the highest hole state, i.e., $0 g_{9 / 2}$.

We will also evaluate a case where no bound singleparticle states are present. For this, we reduced the value of the depth of the Woods-Saxon potential to $V_{0}=37 \mathrm{MeV}$. The corresponding single particle energies are also given in
TABLE I. Values of the Woods-Saxon parameters used in the calculations. The spin-orbit parameters $r_{0}^{s o}$ and $a^{s o}$ coincide with the ones corresponding to the volume part given in this table. The Coulomb radius in the proton cases is the same as $r_{0}$, i.e., $r_{0}^{\text {Coul }}$ $=1.19 \mathrm{fm}$. The meaning of these parameters is as in Ref. [28].

\begin{tabular}{lcccc}
\hline \hline Core & $V_{0}(\mathrm{MeV})$ & $r_{0}(\mathrm{fm})$ & $a(\mathrm{fm})$ & $V_{\text {so }}(\mathrm{MeV})$ \\
\hline${ }^{78} \mathrm{Ni}$ (neutrons) & 40 & 1.27 & 0.67 & 21.43 \\
${ }^{100} \mathrm{Sn}$ (protons) & 58.5 & 1.19 & 0.75 & 15 \\
\hline \hline
\end{tabular}

Table II. Note that even in this case the shell $N=50$ is rather well defined.

\section{Fermi level is bound}

We will first analyze the case where there are bound single-particle states, i.e., the case WS1 in Table II. Already from the start one faces the problem of determining which single-particle states are to be included. In a standard shellmodel calculation one would include the states corresponding to a major shell only plus possibly an intruder state. In our case that would be the shell $N=4$ and the intruder state $0 h_{11 / 2}$. However we now have the imaginary part of the energy to take into account. This may produce states with relatively small real parts of the energies, but very large (in absolute value) imaginary parts. Such states would induce very wide two-particle resonances and their inclusion would imply the use of contours embracing large portions of the complex energy plane which one would not expect to influence the narrow two-particle resonances of interest. We therefore decided to include all single-particle states up to a real energy of $6 \mathrm{MeV}$, and imaginary parts down to $-4 \mathrm{MeV}$. This corresponds to all states shown in Table II.

As mentioned above, to determine the strength of the separable force we will follow the standard procedure of adjusting $G_{\alpha}$ by fitting the energy of a two-particle state, which usually is experimentally known. In our case we will assume that such a state, which would be the ground state of ${ }^{80} \mathrm{Ni}$, exists below twice the energy of the lowest single-particle

TABLE II. Single-particle neutron states in ${ }^{78} \mathrm{Ni}$ evaluated with the Woods-Saxon potential given in Table I. The complex energies are in $\mathrm{MeV}$. The column labeled WS1 corresponds to $V_{0}$ $=40 \mathrm{MeV}$, and WS2 to $V_{0}=37 \mathrm{MeV}$. The hole states $0 g_{9 / 2}$ are given to show the magnitude of the gap corresponding to the magic number $N=50$.

\begin{tabular}{lcc}
\hline \hline State & WS1 & WS2 \\
\hline $0 g_{9 / 2}$ & $(-4.398,0)$ & $(-2.587,0)$ \\
$1 d_{5 / 2}$ & $(-0.800,0)$ & $(0.294,-0.018)$ \\
$2 s_{1 / 2}$ & $(-0.295,0)$ & ----- \\
$1 d_{3 / 2}$ & $(1.325,-0.479)$ & $(1.905,-1.241)$ \\
$0 h_{11 / 2}$ & $(3.296,-0.013)$ & $(4.681,-0.069)$ \\
$1 f_{7 / 2}$ & $(3.937,-1.796)$ & $(4.455,-2.851)$ \\
$0 g_{7 / 2}$ & $(4.200,-0.167)$ & $(5.799,-0.506)$ \\
\hline \hline
\end{tabular}


state, i.e., below $2 \epsilon_{1 d_{5 / 2}}$. This energy gap, i.e., the correlation energy, is more than $1 \mathrm{MeV}$ in well-established normal nuclei, like ${ }^{208} \mathrm{~Pb}$ (where it is $1.244 \mathrm{MeV}$ ) and ${ }^{56} \mathrm{Ni}(1.936$ $\mathrm{MeV})$. However in our case the bound states are so few and so slightly bound that such high-energy gaps do not seem to be reasonable. Since there are not any experimental data which could guide us, and since our intention is just to see how the strength of the force affects the results, we will vary $G_{\alpha}$ from zero to a maximum value corresponding to a gap of $2.527 \mathrm{MeV}$, i.e., for $\mathrm{a}^{80} \mathrm{Ni}$ (g.s.) energy of $-4.183 \mathrm{MeV}$. The value of the strength corresponding to the Berggren basis described below is $G_{\alpha}=0.0028 \mathrm{MeV}$.

In the calculations to be presented here we used a rectangular contour with vertices as in Fig. 4 with $a=0.5 \mathrm{MeV}$, $b=9 \mathrm{MeV}, c=-4 \mathrm{MeV}$, and $d=20 \mathrm{MeV}$. We thus include, in the Berggren basis, all the Gamow states shown in Table II plus the bound states $1 d_{5 / 2}$ and $2 s_{1 / 2}$. With this contour the allowed region comprises a two-particle energy plane with complex energies $\left(E_{r}, E_{i}\right)$, such that $1 \mathrm{MeV}$ $<E_{r}<9 \mathrm{MeV}$ and $-4 \mathrm{MeV}<E_{i}<0 \mathrm{MeV}$.

As already mentioned above, we will use a Gaussian method of integration over the contour. We found that in order to obtain convergence within six digits in the evaluated quantities, one has to include ten Gaussian points for each $\mathrm{MeV}$ on the lines of the contour in Fig. 4, except for the last segment [the one going from $(b, 0)$ to $(d, 0)]$, where five points for each $\mathrm{MeV}$ are enough. We arrive at this conclusion by always choosing the contour such that the resonances lie at least at $300 \mathrm{keV}$ from the borders of the contour. The number of scattering states thus included in the basis is $n_{g}$ $=225$. The convergence of the results as a function of $n_{g}$ as well as the influence of the continuum upon the calculated states will be given below.

One can check the reliability of the results by performing a calculation over the real energy axis only, that is by using the continuum shell model [32]. Any bound state that one may thus obtain should coincide with those evaluated by using any contour. Moreover, when evaluating the strength of the force by given a real energy $\omega_{\alpha}$ in Eq. (7) the value of $G_{\alpha}$ thus obtained should, independent of the contour that one uses, be a real quantity. All these requirements are fulfilled in our calculations. This is important since it is a strong test of the reliability of our computing codes as well as a confirmation of the validity of the formalism.

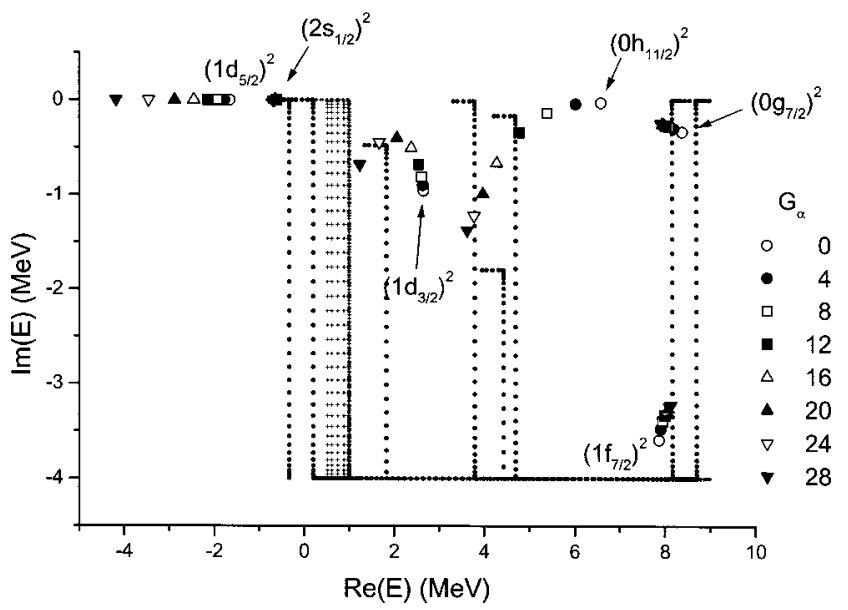

FIG. 7. Energies of the calculated two-particle states as a function of the strength $G_{\alpha}\left(\times 10^{4}\right.$, in MeV) for the case WS1 of Table II. Only the allowed region in the two-particle energy plane is shown. The straight lines formed by small dots correspond to continuum configurations where one particle is in a shell-model state and the other in a scattering state. The crosses correspond to continuum configuration where both particles are in scattering states. The labels of the curves followed by the physical two-particle resonances indicate the corresponding zeroth-order configurations.

In Fig. 7 we show the energies evaluated by using different values of the strength $G_{\alpha}$. The energies follow the pattern discussed in Sec. II. One can thus identify the distinctive straight lines corresponding to scattering configurations. For all $G$ values these lines appear practically in the same position. One can also see that the two-particle resonances can readily be distinguished from the scattering states by just looking at the figure. This is possibly due to the presence of an allowed region which only contains the physical states.

In the figure the physical states are labeled by their zeroth-order configurations. One sees that as the interaction increases the real part of the energies behave in a standard shell-model fashion. Thus the state with the largest degeneracy "feels" the interaction the most. Moreover, the ground state departs from the rest of the spectrum more and more as the interaction increases, also in agreement with expectations. Guided by these well-established facts one may assume that narrow configurations, which due to the centrifugal barrier are usually the ones with highest degeneracy, would follow a similar pattern. That is, they would be domi-

TABLE III. Main components of the wave functions corresponding to the state which in zeroth order is $\left(0 h_{11 / 2}\right)^{2}$ as a function of $G_{\alpha}\left(\times 10^{4}\right.$, in MeV) for the case WS1 of Table II. The corresponding two-particle energy $E$ (in $\mathrm{MeV}$ ) is also given. Only components which in absolute value are larger than 0.14 are given. The basis states are ordered according to their widths. Thus $\left(0 h_{11 / 2}\right)^{2}$ is the narrowest and $\left(1 f_{7 / 2}\right)^{2}$ the widest configuration.

\begin{tabular}{lcccccc}
\hline \hline$G_{\alpha}$ & $E$ & $\left(0 h_{11 / 2}\right)^{2}$ & $\left(0 g_{7 / 2}\right)^{2}$ & $\left(1 d_{3 / 2}\right)^{2}$ & $\left(1 f_{7 / 2}\right)^{2}$ & $\left(1 d_{5 / 2}\right)^{2}$ \\
\hline 8 & $(5.399,-0.136)$ & $(0.96,0.00)$ & $(-0.27,-0.02)$ & - & $(0.14,0.02)$ & - \\
12 & $(4.784,-0.342)$ & $(0.92,0.02)$ & $(-0.33,-0.03)$ & $(0.14,0.02)$ & $(0.20,0.03)$ & - \\
16 & $(4.283,-0.664)$ & $(0.86,0.05)$ & $(-0.35,-0.05)$ & $(0.36,-0.19)$ & $(0.23,0.03)$ & $(0.14,0.07)$ \\
20 & $(3.975,-0.990)$ & $(0.77,0.04)$ & $(-0.33,-0.06)$ & $(0.52,-0.11)$ & $(0.24,0.03)$ & $(0.14,0.10)$ \\
24 & $(3.780,-1.222)$ & $(0.70,-0.01)$ & $(-0.32,-0.03)$ & $(0.60,-0.01)$ & $(0.24,0.01)$ & $(0.15,0.10)$ \\
28 & $(3.628,-1.376)$ & $(0.66,-0.06)$ & $(-0.31,-0.01)$ & $(0.66,0.06)$ & $(0.23,-0.01)$ & $(0.16,0.10)$ \\
\hline \hline
\end{tabular}


TABLE IV. As in Table III for the state which in zeroth order is $\left(1 d_{3 / 2}\right)^{2}$. The relevant basis states (i.e., with amplitudes larger than 0.2) include now the bound configuration $\left(1 d_{5 / 2}\right)^{2}$ and configurations consisting of scattering states, of which we give only the largest component under the column "scat."

\begin{tabular}{lccccccc}
\hline \hline$G_{\alpha}$ & $E$ & $\left(1 d_{5 / 2}\right)^{2}$ & $\left(0 h_{11 / 2}\right)^{2}$ & $\left(0 g_{7 / 2}\right)^{2}$ & $\left(1 d_{3 / 2}\right)^{2}$ & $\left(1 f_{7 / 2}\right)^{2}$ & scat \\
\hline 8 & $(2.604,-0.809)$ & - & - & - & $(1.00,0.01)$ & - & - \\
12 & $(2.541,-0.679)$ & - & - & - & $(0.99,0.03)$ & - & - \\
16 & $(2.378,-0.506)$ & - & $(-0.33,0.15)$ & - & $(0.94,0.07)$ & - & - \\
20 & $(2.059,-0.401)$ & $(-0.25,-0.02)$ & $(-0.46,0.06)$ & $(0.23,0.04)$ & $(0.88,0.05)$ & - & - \\
24 & $(1.665,-0.447)$ & $(-0.31,-0.08)$ & $(-0.48,-0.00)$ & $(0.25,-0.00)$ & $(0.70,-0.05)$ & - & - \\
28 & $(1.235,-0.683)$ & $(-0.46,-0.22)$ & $(-0.63,-0.04)$ & $(0.33,0.01)$ & $(0.74,-0.19)$ & $(-0.24,0.06)$ & - \\
\hline \hline
\end{tabular}

nant in building up the physical (narrow) two-particle resonances, as assumed in Refs. [14-16]. But Fig. 7 shows just the opposite situation. As the interaction increases all resonances become narrower, except the one corresponding to the configuration $\left(0 h_{11 / 2}\right)^{2}$, which becomes wider. This unexpected feature is a consequence of the Berggren metric, i.e. of the non-Hermitian character of the Hamiltonian matrix in the complex energy sector. Similar properties, like violations of the noncrossing of levels rule, were found in the oneparticle case [33].

The behavior of the physical resonances in Fig. 7 is rather involved. Thus the states labeled $\left(1 d_{3 / 2}\right)^{2}$ first become narrower as the interaction increases, but, at a certain point, for $G_{\alpha}=0.002 \mathrm{MeV}$ in the figure, this tendency is reversed. At the same time the increase of $-\operatorname{Im}(E)$ desaccelerates around the same value of $G_{\alpha}$ for the states $\left(0 h_{11 / 2}\right)^{2}$.

To understand the behavior of the states $\left(0 h_{11 / 2}\right)^{2}$, in Table III we give the main components of the corresponding wave functions. A feature to be noticed is that in no case are bound configurations relevant. As the interaction increases wide configurations become more and more important. This explains why the state becomes wider. However, the bound configuration $\left(1 d_{5 / 2}\right)^{2}$ start to become relevant at a large enough value of $G_{\alpha}$. Thus, at $G_{\alpha}=0.002 \mathrm{MeV}$, that configuration contributes a value of $(0.14,0.007)$ to the wave function. This value becomes more important as $G_{\alpha}$ becomes larger, thus desaccelerating the increase of the widths.

On the other hand, the wave functions of the states $\left(1 d_{3 / 2}\right)^{2}$ presented in Table IV show that narrow configurations [more exactly, configurations narrower than the zerothorder one, which here is $\left.\left(1 d_{3 / 2}\right)^{2}\right]$ contribute substantially to the structure of the state as the interaction increases, particularly the narrow states $\left(0 h_{11 / 2}\right)^{2}$ and $\left(0 g_{7 / 2}\right)^{2}$ and the bound configuration $\left(1 d_{5 / 2}\right)^{2}$. This explains why the states $\left(1 d_{3 / 2}\right)^{2}$ become narrower as a function of $G_{\alpha}$. But as $G_{\alpha}$ increases scattering configurations become important and, as a result, the states become wider. An interesting feature in this contex is the sudden appearance of a large contribution [of a value $(0.42,0.18)]$ from a scattering configuration at $G_{\alpha}$ $=0.0024 \mathrm{MeV}$. This corresponds to the configuration $|C\rangle$ $=\left|0 d_{3 / 2} c_{3 / 2}\right\rangle$, where $c_{3 / 2}$ is the scattering function at $(0.385,0) \mathrm{MeV}$. This is a Gaussian point on the first border of the contour. The energy of the configuration $|C\rangle$ is (in $\mathrm{MeV})(1.325,-0.479)+(0.385,0)=(1.710,-0.479)$, which is very close to the energy of the resonance, i.e., $(1.665,-0.447)$. This can even be inferred from Fig. 7 where the down open triangle for the case $\left(1 d_{3 / 2}\right)^{2}$ being discussed here practically overlaps with our continuum configuration $|C\rangle$. Therefore, according to Eq. (8), the corresponding wave function component is large.

The unexpected behavior of the resonances discussed above is representative of all the others in Fig. 7, while the bound states behave in a standard shell-model fashion. It is perhaps surprising that the first excited bound states [labeled $\left(2 s_{1 / 2}\right)^{2}$ in the figure] do not show any remarkable sensitivity to scattering states, although they lie close to the continuum threshold. Indeed, the wave functions corresponding to these states consist mainly of the configurations $\left(1 d_{5 / 2}\right)^{2}$ and $\left(2 s_{1 / 2}\right)^{2}$ for all values of $G_{\alpha}$.

One important feature of the calculation is that the energies corresponding to physical states converge to their exact values relatively fast as a function of the dimension of the basis. We show this for the states $\left(1 d_{3 / 2}\right)^{2}$ and $\left(0 h_{11 / 2}\right)^{2}$ in Tables V and VI, respectively. To assess whether the strength of the interaction affects the convergence, we have chosen different values of $G_{\alpha}$. We thus see that indeed the energy

TABLE V. Convergence of energies corresponding to the states labeled $\left(1 d_{3 / 2}\right)^{2}$ in Fig. 7 as a function of the number of Gaussian points $n_{g}$. The value $n_{g}=0$ corresponds to the case where only bound states and Gamow resonances are included in the basis. The columns are labeled by the strength $G_{\alpha}\left(\times 10^{4}\right.$, in $\left.\mathrm{MeV}\right)$.

\begin{tabular}{lcccc}
\hline \hline$n_{g}$ & 4 & 20 & 24 & 28 \\
\hline 0 & $(2.640,-0.896)$ & $(3.299,-0.607)$ & $(3.275,-0.858)$ & $(3.227,-0.975)$ \\
10 & $(2.63416,-0.89697)$ & $(2.13004,-0.42801)$ & $(1.79169,-0.50527)$ & $(1.360,-0.82097)$ \\
50 & $(2.63448,-0.89643)$ & $(2.05694,-0.39779)$ & $(1.70880,-0.43585)$ & $(1.24293,-0.68603)$ \\
100 & $(2.63349,-0.89643)$ & $(2.05889,-0.40198)$ & $(1.67618,-0.44027)$ & $(1.23509,-0.68299)$ \\
150 & $(2.63349,-0.89643)$ & $(2.05889,-0.40198)$ & $(1.67618,-0.44027)$ & $(1.23509,-0.68299)$ \\
\hline \hline
\end{tabular}


TABLE VI. As in Table V for the states $\left(0 h_{11 / 2}\right)^{2}$.

\begin{tabular}{lcccc}
\hline \hline$n_{g}$ & \multicolumn{1}{c}{4} & 20 & 24 & 28 \\
\hline 0 & $(6.018,0.004)$ & $(2.777,0.320)$ & $(2.237,0.681)$ & $(1.775,0.860)$ \\
10 & $(6.02747,-0.03903)$ & $(3.95235,-0.95715)$ & $(3.75050,-1.18517)$ & $(3.59543,-1.33198)$ \\
50 & $(6.02693,-0.03949)$ & $(3.97506,-0.98989)$ & $(3.77995,-1.22224)$ & $(3.62815,-1.37649)$ \\
100 & $(6.02693,-0.03949)$ & $(3.97507,-0.98988)$ & $(3.77989,-1.22213)$ & $(3.62798,-1.37641)$ \\
150 & $(6.02693,-0.03949)$ & $(3.97507,-0.98988)$ & $(3.77989,-1.22213)$ & $(3.62798,-1.37641)$ \\
\hline \hline
\end{tabular}

corresponding to $G_{\alpha}=0.0004 \mathrm{MeV}$, which is the smallest $G$ value shown in those tables, coincides within a few $\mathrm{keV}$ with the exact result already for $n_{g}=0$. But as $G_{\alpha}$ is increased that agreement deteriorates. Particularly inadequate are the energies evaluated by using $n_{g}=0$ for the states $\left(0 h_{11 / 2}\right)^{2}$ and $G_{\alpha} \geqslant 0.0020 \mathrm{MeV}$. Not only are the real parts of those energies wrong by an amount ranging from $1.2 \mathrm{Mev}$ (for $G_{\alpha}=0.0020 \mathrm{MeV}$ ) to almost $2 \mathrm{MeV}$, but also the imaginary parts are large and positive, which does not make sense since it would correspond, e.g., to negative widths. This last feature does not appear for the states $\left(1 d_{3 / 2}\right)^{2}$.

One can understand the deterioration of the resonant shell model results (i.e., of neglecting the continuum by using $n_{g}$ $=0)$ as the strength increases by noticing that it is through the interaction that continuum configurations become relevant in the calculation. This also explains why the results corresponding to $n_{g}=0$ for the states $\left(1 d_{3 / 2}\right)^{2}$ are generally better than those corresponding to $\left(0 h_{11 / 2}\right)^{2}$, since here the interaction is stronger (due to the degeneracy) for a given value of $G_{\alpha}$. But, already with $n_{g}=10$, the agreement between the exact results and the approximated ones is reasonable in all these cases of physical states. Moreover, for $n_{g}$ $=100$ the exact results are reproduced within six digits. This convergence is better than the one required to achieve a similar agreement in general, for which one needs the value $n_{g}$ $=225$ used in our calculations, as mentioned above.

Finally it is worthwhile to point out that the presence of scattering states lying near to physical states does not affect the convergence, as seen in, e.g., Table $\mathrm{V}$ for the state $\left(1 d_{3 / 2}\right)^{2}$ with $G_{\alpha}=0.0024 \mathrm{MeV}$ (cf. Fig. 7).

\section{Fermi level is unbound}

In this subsection we will analyze the case where there is not any bound single-particle states, i.e., the case WS2 in Table II. Actually there is not any essential difference between this case and the previous one since within this formalism all states (including the continuum states) are treated on the same footing, independently of the location of the Fermi level.

The single-particle resonances are wider than before, and therefore we used here a different one-particle contour, namely $a=0.1 \mathrm{MeV}, b=13 \mathrm{MeV}, c=-6 \mathrm{MeV}$, and $d$ $=26 \mathrm{MeV}$.

In Fig. 8 we present the evaluated states as a function of the strength $G_{\alpha}$, which we allowed to vary within the same range as in the previous case. The straight lines discussed above appear also in this case, with the same characteristics as before.
Even the physical resonances present the same features as in Sec. III A 1. In particular, the states labeled $\left(0 h_{11 / 2}\right)^{2}$ interact strongly with all the others thereby becoming wider while all the other states become narrower.

However, there is an important feature in this case, namely, the development of a bound state which is induced by the two-particle interaction, as shown by the states $\left(1 d_{5 / 2}\right)^{2}$. To analyze the reason for this behavior, in Table VII we present the main components of the corresponding wave functions. As expected, one sees that when the interaction is weak the state is built practically by the configuration $\left(1 d_{5 / 2}\right)^{2}$ only. As $G_{\alpha}$ increases the two-particle resonance approaches the continuum threshold, and scattering states contribute substantially to the wave function. Thus the state under the column labeled scat corresponding to $G_{\alpha}$ $=0.0012 \mathrm{MeV}$ is $1 d_{5 / 2} c_{5 / 2}$, where $c_{5 / 2}$ is a scattering $d$ wave at an energy $(0.089,0) \mathrm{MeV}$. At $G_{\alpha}=0.0020 \mathrm{MeV}$ the resonance approaches threshold even more and here the continuum itself becomes important. Indeed, the large contribution under the column scat now corresponds to the configuration $c_{1 / 2} c_{1 / 2}$, where $c_{1 / 2}$ is a scattering $s$ wave with an energy $(0.011,0) \mathrm{MeV}$, itself very close to threshold. As the interaction increases even more the state becomes bound,

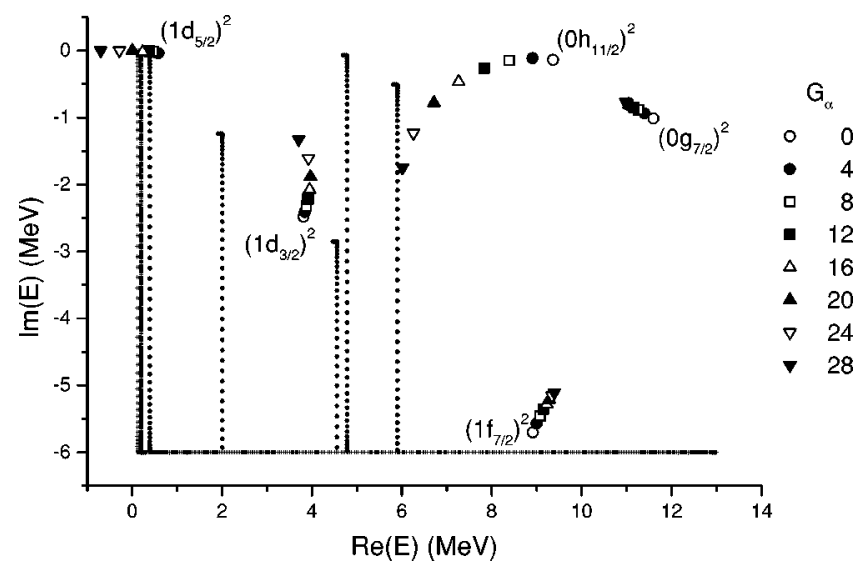

FIG. 8. Energies of the calculated two-particle states as a function of the strength $G_{\alpha}\left(\times 10^{4}\right.$, in MeV) for the case WS2 of Table II. Only the allowed region in the two-particle energy plane is shown. The straight lines consisting of small dots correspond to continuum configurations where one particle is in a bound or Gamow state and the other in a scattering state. The crosses correspond to continuum configuration where both particles are in scattering states. The labels of the curves followed by the physical two-particle resonances indicate the corresponding zeroth-order configurations. 
TABLE VII. Main components of the wave functions corresponding to the state which in zeroth order is $\left(1 d_{5 / 2}\right)^{2}$ as a function of $G_{\alpha}\left(\times 10^{4}\right.$, in $\left.\mathrm{MeV}\right)$ for the case WS2 of Table II. Under the column "scat" we give the largest component corresponding to configurations consisting of scattering states. The two-particle energy $E$ (in $\mathrm{MeV}$ ) is also given. Only components which in absolute value are larger than 0.2 are given.

\begin{tabular}{lcccccc}
\hline \hline$G_{\alpha}$ & $E$ & $\left(1 d_{5 / 2}\right)^{2}$ & $\left(0 h_{11 / 2}\right)^{2}$ & $\left(0 g_{7 / 2}\right)^{2}$ & $\left(1 d_{3 / 2}\right)^{2}$ & scat \\
\hline 4 & $(0.538,-0.024)$ & $(1.00,0.00)$ & - & - & - & - \\
12 & $(0.377,-0.010)$ & $(1.00,-0.04)$ & - & - & - & - \\
20 & $0.002,-0.000$ & $(0.81,-0.04)$ & - & - & - & - \\
28 & $(-0.700,0)$ & $(0.84,-0.04)$ & $(-0.32,-0.23)$ \\
\hline \hline
\end{tabular}

and at $G_{\alpha}=0.0028 \mathrm{MeV}$ the scattering states cease to be important. But the interaction is strong enough here to mix up all the shell-model configurations, showing the importance of Gamow resonances in inducing bound states in nuclei that lie far from the line of $\beta$ stability.

\section{B. Two-proton resonances}

Proton resonances are usually narrower than the corresponding neutron ones due to the Coulomb barrier. It is therefore often in this case that one studies many-body systems including only narrow Gamow resonances. In this section we analyze this approximation for the case of two protons outside the ${ }^{100} \mathrm{Sn}$ core. The single-particle proton states correspond to the major shell $N=4$, which is the same as in the previous subsections. The core mean field is described by a Wood-Saxon potential with the parameters given in Table I. These parameters were adjusted to obtain the single-particle states shown in Table VIII, which agree with systematics in this region. Notice that none of these single-particle states is bound.

As in the neutron case analyzed above, we include in our single-particle representation even states which belong to higher shells, namely, states $1 f_{7 / 2}$ and $0 i_{13 / 2}$, because they are relatively narrow. We include these high-lying shells in order to assess whether they can be neglected, as one does within the standard shell model.

We chose even here the rectangular contour of Fig. 4 with vertices defined by the values $a=0.1 \mathrm{MeV}, b=19 \mathrm{MeV}, c$ $=-1 \mathrm{MeV}$, and $d=26 \mathrm{MeV}$. This contour encloses all the Gamow resonances of Table VIII. Choosing the Gaussian points as indicated above in order to obtain a precision of six

TABLE VIII. Single-particle proton states in ${ }^{100} \mathrm{Sn}$ evaluated with the Woods-Saxon potential given in Table I. The complex energies are in $\mathrm{MeV}$.

\begin{tabular}{lc}
\hline \hline State & Energy \\
\hline $1 d_{5 / 2}$ & $(2.583,-0.000)$ \\
$2 s_{1 / 2}$ & $(4.007,-0.004)$ \\
$0 g_{7 / 2}$ & $(4.469,-0.000)$ \\
$1 d_{3 / 2}$ & $(4.917,-0.004)$ \\
$0 h_{11 / 2}$ & $(7.559,-0.001)$ \\
$1 f_{7 / 2}$ & $(9.710,-0.424)$ \\
$0 i_{13 / 2}$ & $(16.361,-0.210)$ \\
\hline \hline
\end{tabular}

digits, the number of scattering states for each partial wave turns out to be $n_{g}=298$.

With the single-particle (Berggren) representation thus established we calculated the complex two-particle energies by solving the dispersion relation [Eq. (7)]. The corresponding wave functions were evaluated by using Eq. (8).

We used in our calculations of the two-proton states, which would be resonances in ${ }^{102} \mathrm{Te}$, values of the strength $G_{\alpha}$ in a range similar to that in the neutron cases analyzed in the previous subsections. The results of the calculation are shown in Fig. 9. The general trends in this figure are similar to the ones already found for the two-neutron cases.

One notices that even in this case where all resonances are very narrow, the narrowest resonance in zeroth order becomes wider as the interaction increases, while all the others become narrower. This is especially remarkable for the state that at zeroth order is $\left(2 s_{1 / 2}\right)^{2}$, since one does not expect that a state with such low degeneracy would be important in building up low-lying resonances. To analyze these states, in Table IX we present the corresponding wave-function amplitudes for values of the strength $G_{\alpha}$ used in Fig. 9. As ex-

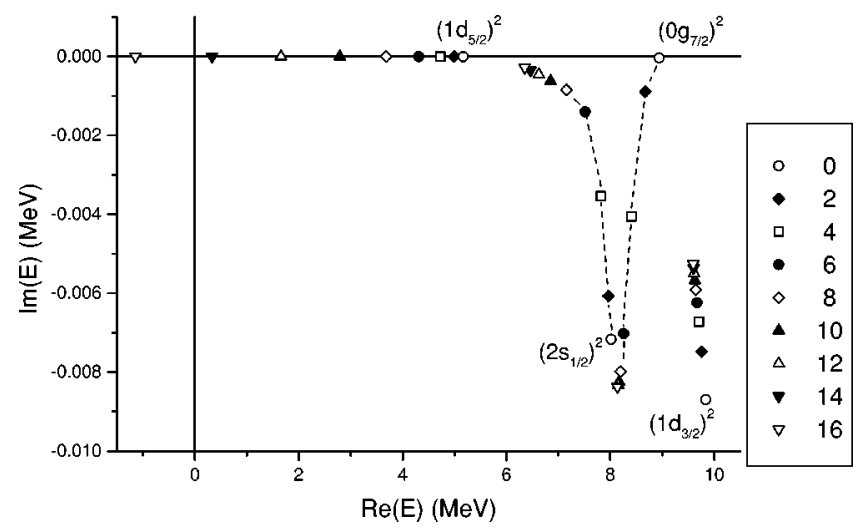

FIG. 9. Energies of the physical two-particle states calculated as a function of the strength $G_{\alpha}\left(\times 10^{4}\right.$, in $\left.\mathrm{MeV}\right)$ for the proton case of Table VIII. All physical resonances lying up to an energy of 10 $\mathrm{MeV}$ are shown. Notice the scale in the imaginary part of the energy, which indicates that the widths of the physical resonances are in all cases small. The labels of the curves followed by the physical two-particle resonances indicate the corresponding zeroth-order configurations. The dashed lines were drawn to guide the eye. 
TABLE IX. Main components of the two-proton wave functions corresponding to the state which in zeroth order is $\left(2 s_{1 / 2}\right)^{2}$ in Fig. 9 as a function of $G_{\alpha}\left(\times 10^{4}\right.$, in $\left.\mathrm{MeV}\right)$. The single-particle states are as in Table VIII. Only components which in absolute value are larger than 0.2 are given.

\begin{tabular}{lccccc}
\hline \hline$G_{\alpha}$ & $\left(1 d_{5 / 2}\right)^{2}$ & $\left(2 s_{1 / 2}\right)^{2}$ & $\left(0 g_{7 / 2}\right)^{2}$ & $\left(1 d_{3 / 2}\right)^{2}$ & $\left(0 h_{11 / 2}\right)^{2}$ \\
\hline 2 & - & $(0.99,0.00)$ & - & - & - \\
6 & $(-0.28,0.00)$ & $(0.67,0.00)$ & $(0.61,-0.00)$ & $(0.23,-0.00)$ & - \\
10 & $(-0.58,-0.00)$ & $(0.42,-0.00)$ & $(0.60,0.00)$ & $(0.26,-0.00)$ & $(-0.22,0.00)$ \\
12 & $(-0.68,-0.00)$ & $(0.35,-0.00)$ & $(0.54,0.00)$ & $(0.25,-0.00)$ & $(-0.22,0.00)$ \\
14 & $(-0.74,-0.00)$ & $(0.31,-0.00)$ & $(0.50,0.00)$ & $(0.23,-0.00)$ & $(-0.21,0.00)$ \\
16 & $(-0.78,-0.00)$ & $(0.28,-0.00)$ & $(0.46,0.00)$ & $(0.22,-0.00)$ & $(-0.20,0.00)$ \\
\hline \hline
\end{tabular}

TABLE X. Main components of the two-proton wave functions corresponding to the state which in zeroth order is $\left(1 d_{5 / 2}\right)^{2}$ in Fig. 9 as a function of $G_{\alpha}\left(\times 10^{4}\right.$, in MeV). The single-particle states are as in Table VIII. Only components which in absolute value are larger than 0.2 are given.

\begin{tabular}{lcccccc}
\hline \hline$G_{\alpha}$ & $\left(1 d_{5 / 2}\right)^{2}$ & $\left(2 s_{1 / 2}\right)^{2}$ & $\left(0 g_{7 / 2}\right)^{2}$ & $\left(1 d_{3 / 2}\right)^{2}$ & $\left(0 h_{11 / 2}\right)^{2}$ & $\left(0 i_{13 / 2}\right)^{2}$ \\
\hline 2 & $(1.00,0.00)$ & - & - & - & - & - \\
6 & $(0.95,0.00)$ & - & $(0.23,0.00)$ & - & - & - \\
10 & $(0.80,0.00)$ & - & $(0.40,0.00)$ & $(0.22,-0.00)$ & $(-0.29,-0.00)$ & - \\
12 & $(0.72,0.00)$ & $(0.20,-0.00)$ & $(0.45,0.00)$ & $(0.25,-0.00)$ & $(-0.35,-0.00)$ & - \\
14 & $(0.65,0.00)$ & $(0.21,-0.00)$ & $(0.47,0.00)$ & $(0.27,-0.00)$ & $(-0.40,-0.00)$ & $(0.20,-0.01)$ \\
16 & $(0.60,0.00)$ & $(0.21,-0.00)$ & $(0.48,0.00)$ & $(0.28,-0.00)$ & $(-0.43,-0.00)$ & $(0.23,-0.01)$ \\
\hline \hline
\end{tabular}

TABLE XI. Convergence of energies corresponding to the states labeled $\left(1 d_{5 / 2}\right)^{2}$ in Fig. 9 as a function of the number of Gaussian points $n_{g}$. The columns are labeled by the strength $G_{\alpha}\left(\times 10^{4}\right.$, in $\left.\mathrm{MeV}\right)$.

\begin{tabular}{lcccc}
\hline \hline$n_{g}$ & 2 & 10 & 14 & 16 \\
\hline 0 & $(4.996,0.000)$ & $(3.118,0.142)$ & $(1.150,0.349)$ & $(-0.030,0.474)$ \\
10 & $(4.99275,0.00151)$ & $(2.76320,0.16744)$ & $(0.27597,0.41367)$ & $(-1.21486,0.56018)$ \\
50 & $(4.99316,0.00007)$ & $(2.79745,0.00742)$ & $(0.35026,0.01761)$ & $(-1.12083,0.02337)$ \\
100 & $(4.99302,-0.00000)$ & $(2.79031,-0.00017)$ & $(0.33314,-0.00040)$ & $(-1.14366,-0.00053)$ \\
150 & $(4.99309,-0.00000)$ & $(2.79025,-0.00000)$ & $(0.33299,-0.00000)$ & $(-1.14386,-0.00000)$ \\
200 & $(4.99309,-0.00000)$ & $(2.79025,-0.00000)$ & $(0.33299,-0.00000)$ & $(-1.14386,-0.00000)$ \\
\hline \hline
\end{tabular}

TABLE XII. Two-proton wave-function amplitudes corresponding to $G_{\alpha}=0.0016 \mathrm{MeV}$ in Table XI as a function of the number of scattering states $n_{g}$ included in the basis. Only components which in absolute value are larger than 0.2 are given.

\begin{tabular}{lcccccc}
\hline \hline$n_{g}$ & $\left(1 d_{5 / 2}\right)^{2}$ & $\left(0 g_{7 / 2}\right)^{2}$ & $\left(0 h_{11 / 2}\right)^{2}$ & $\left(1 d_{3 / 2}\right)^{2}$ & $\left(0 i_{13 / 2}\right)^{2}$ & $\left(2 s_{1 / 2}\right)^{2}$ \\
\hline 0 & $(0.641,0.025)$ & $(0.478,0.000)$ & $(-0.413,0.009)$ & $(0.274,-0.003)$ & $(0.212,-0.022)$ & $(0.208,-0.000)$ \\
10 & $(0.592,0.023)$ & $(0.479,0.003)$ & $(-0.435,0.007)$ & $(0.277,-0.002)$ & $(0.232,-0.023)$ & $(0.206,0.000)$ \\
50 & $(0.598,0.004)$ & $(0.480,0.003)$ & $(-0.433,-0.001)$ & $(0.277,-0.000)$ & $(0.231,-0.014)$ & $(0.206,0.000)$ \\
100 & $(0.598,0.004)$ & $(0.480,0.003)$ & $(-0.433,-0.001)$ & $(0.277,-0.000)$ & $(0.231,-0.014)$ & $(0.206,0.000)$ \\
\hline \hline
\end{tabular}


pected according to what we found for the neutron cases above, the reason these states become narrower is that narrow configurations play an important role as the interaction increases. However, this trend is not as specific as before, when just the narrowest neutron configuration [which was $\left.\left(0 h_{11 / 2}\right)^{2}\right]$ contributed most to the narrowing wave function. The equivalent shell is now $\left(0 g_{7 / 2}\right)^{2}$, which first (at low values of $G_{\alpha}$ ) is important but then decreases as the strength increases. Perhaps even more amazing is the behavior of the shell $\left(1 d_{3 / 2}\right)^{2}$, which first increases in importance but suddenly, starting at $G_{\alpha}=0.0010 \mathrm{MeV}$, decreases again. These features again indicate that the behavior of the wave functions in the Berggren space can follow patterns which are unusual from a standard shell-model viewpoint. The only configuration in Table IX which increases continuously in absolute value as $G_{\alpha}$ increases is $\left(1 d_{5 / 2}\right)^{2}$, which is also very narrow, and which may explain why these two-proton resonances become narrower.

The other notable states in Fig. 9 are those labeled $\left(1 d_{5 / 2}\right)^{2}$, which are very narrow for all values of $G_{\alpha}$ and which rapidly decrease in energy as $G_{\alpha}$ increases, as expected for a pairing (ground) excitation. Eventually the state becomes bound for a large enough value of the strength, which in the figure is between 0.0014 and $0.0016 \mathrm{MeV}$. To study the changing structure of these pairing states, in Table $\mathrm{X}$ we show the corresponding amplitudes as a function of $G_{\alpha}$. As expected from true pairing vibrations [34], the number of equally important configurations increases with the strength of the pairing force. Moreover, the real parts of the wave-function components (which actually are virtually real numbers) carry the phase $(-1)^{l}$, where $l$ is the orbital angular momentum of the corresponding single-particle states. In this subject of pairing vibrations the results of the method presented here and those of the standard shell-model coincide.

In this case of very narrow Gamow resonances one notices that in the two-particle wave functions the scattering states do not seem to play an important role (cf. Table IV). To analyze this point, in Table XI we present the dependence of the calculated energies, also for the states labeled $\left(1 d_{5 / 2}\right)^{2}$ in Fig. 9, upon the number of scattering states $n_{g}$ included in the Berggren basis. The general features of the results in this table do not differ much from those found in Tables $\mathrm{V}$ and VI. That is, for small values of the strength $G_{\alpha}$ the evaluated energies reach than exact values quickly as $n_{g}$ increases. But this convergence wanes as $G_{\alpha}$ increases. Thus the energy evaluated by neglecting the scattering states agrees with the exact results within a few $\mathrm{keV}$ for $G_{\alpha}=0.0002 \mathrm{MeV}$, but disagrees strongly for $G_{\alpha}=0.0016 \mathrm{MeV}$.

It is interesting to see whether the corresponding wave functions converge as badly as the energies do for large values of the strength. We show this in Table XII, where we use the extreme case $G_{\alpha}=0.0016 \mathrm{MeV}$. Perhaps surprisingly, one sees that the main components of the wave functions evaluated for $n_{g}=0$ agrees within a few percent with the exact ones. This shows that the use of only narrow Gamow resonances, neglecting the continuum as done in Ref. [16], may be appropriate to evaluate wave functions although the energies thus obtained are inadequate.

\section{SUMMARY AND CONCLUSIONS}

In this paper we have presented a formalism to evaluate two-particle resonances microscopically within the Berggren representation. This consists of bound states, Gamow resonances, and an infinite (continuous) set of complex scattering states lying on a contour in the complex one-particle energy plane. The Gamow states included in the representation are those enclosed by the contour. The scattering states appear as an integral over the contour. We discretized this integral by using a Gaussian integration procedure. Therefore, the infinite set of scattering states becomes reduced to the finite value $n_{g}$ of Gaussian points. Using this finite Berggren basis we constructed a two-particle basis set of states as the tensorial product of the one-particle basis with itself, as in standard shell-model calculations. We have shown that using an arbitrary contour one may get a two-particle basis with energies covering the whole two-particle complex energy plane of interest. This would hinder the evaluation of two-particle states, since they would be embeded in a continuous set of basis states. To avoid this drawback we have shown that there exists a contour that leaves a region in the two-particle complex energy plane free of basis states. It is just in this region where the physically relevant resonances lie. Using this contour we have evaluated all two-particle resonances with a precision of six digits by choosing $n_{g}$ with values between 150 and 300, depending upon the case under study. But we have found that with $n_{g} \approx 10$ one obtains a precision of a few $\mathrm{keV}$ for the energies of the relevant resonances, while the corresponding wave functions are provided within a precision of a few percent by neglecting the scattering states altogether, i.e., with $n_{g}=0$.

We have applied the formalism to study neutron excitations $\mathrm{in}^{78} \mathrm{Ni}$ and proton excitations in ${ }^{100} \mathrm{Sn}$. The singleparticle states were provided by a Woods-Saxon potential, and we chose a separable force as the two-particle effective interaction.

For the neutron case we analyzed a case where the Fermi level was bound and another one where it lied in the continuum. In both cases wide resonances were included in the basis. For the proton case the Fermi level also lied in the continuum, but here all Gamow resonances were narrow. We have shown that the position of the Fermi level is irrelevant, since all basis states are treated on the same footing.

We have shown that states which in zeroth order consist of configurations containing scattering states feel the interaction very weakly. Instead, the physical states consist mainly of configurations containing only bound states and Gamow resonances. These configurations are the ones expected from the shell model. Even in cases where no bound configurations are present, the two-body interaction may induce narrow resonances and bound two-particle states. We found that the narrowest of those configurations in zeroth order become wider as the interaction increases. At the same time, all the other states become narrower. This unexpected result, which is induced by the Berggren metric, shows that physically relevant resonances, i.e., narrow ones, may be strongly influ- 
enced by states lying deep in the continuum. Although the wave functions of the physical two-particle resonances are mainly built upon shell-model configurations, the corresponding energies are strongly influenced by scattering states. Finally, it is important to point out that the application of the method presented here shows that it is a natural generalization of the shell model to the complex energy plane.

\section{ACKNOWLEDGMENTS}

This work was supported by FOMEC (Argentina), by the Hungarian OTKA fund Nos. T26244, T37991, and T29003, by the Swedish Foundation for International Cooperation in Research and Higher Education (STINT), and by the Swedish Institute.
[1] H. Feshbach, Ann. Phys. (N.Y.) 5, 357 (1958).

[2] M.V. Stoitsov, J. Dobaczewski, P. Ring, and S. Pittel, Phys. Rev. C 61, 034311 (2000).

[3] D.S. Delion and R.J. Liotta, Phys. Rev. C 54, 292 (1996).

[4] G.A. Gamow, Z. Phys. 51, 204 (1928).

[5] A.F.J. Siegert, Phys. Rev. 56, 750 (1939).

[6] T. Berggren, Nucl. Phys. A109, 265 (1968).

[7] W.J. Romo, Nucl. Phys. A116, 617 (1968).

[8] Y.B. Zel'dovich, Zh. Éksp. Teor. Fiz. 39, 766 (1960) [Sov. Phys. JETP 12, 542 (1961)].

[9] B. Gyarmati and T. Vertse, Nucl. Phys. A160, 573 (1971).

[10] T. Berggren and P. Lind, Phys. Rev. C 47, 768 (1993).

[11] R.J. Liotta, E. Maglione, N. Sandulescu, and T. Vertse, Phys. Lett. B 367, 1 (1996).

[12] R. Id Betan, R.J. Liotta, N. Sandulescu, and T. Vertse, Phys. Rev. Lett. 89, 042501 (2002).

[13] N. Michel, W. Nazarewicz, M. Ploszajczak, and K. Bennaceur, Phys. Rev. Lett. 89, 042502 (2002).

[14] V.I. Goldansky, Nucl. Phys. 19, 482 (1960).

[15] V.M. Galitsky and V.F. Cheltsov, Nucl. Phys. 56, 86 (1964).

[16] O. Civitarese, R.J. Liotta, and T. Vertse, Phys. Rev. C 64, 057305 (2001).

[17] A. Bianchini, R.J. Liotta, and N. Sandulescu, Phys. Rev. C 63, 024610 (2001).

[18] A.A. Sonzogni et al., Phys. Rev. Lett. 83, 1116 (1999).

[19] L.S. Ferreira, E. Maglione, and D. Fernandes, Phys. Rev. C 65,
024323 (2002), and references therein.

[20] F. Soramel et al., Phys. Rev. C 63, 031304(R) (2001).

[21] A.T. Kruppa, B. Barmore, W. Nazarewicz, and T. Vertse, Phys. Rev. Lett. 84, 4549 (2000), and references therein.

[22] R.G. Lovas et al., Phys. Rep. 294, 265 (1998).

[23] T. Vertse, R.J. Liotta, and E. Maglione, Nucl. Phys. A584, 13 (1995).

[24] G. Colo et al., Phys. Lett. B 276, 279 (1992).

[25] P. Curutchet, T. Vertse, and R.J. Liotta, Phys. Rev. C 39, 1020 (1989).

[26] H. Esbensen, G.F. Bertsch, and K. Hencken, Phys. Rev. C 56, 3054 (1997), and references therein.

[27] M.V. Zhukov et al., Phys. Rep. 231, 151 (1993).

[28] T. Vertse, K.F. Pál, and Z. Balogh, Comput. Phys. Commun. 27, 309 (1982).

[29] L. Gr. Ixaru, Numerical Methods for Differential Equations (Reidel, Dordrecht, 1984); L. Gr. Ixaru, M. Rizea, and T. Vertse, Comput. Phys. Commun. 85, 217 (1995).

[30] A.M. Lane, Nuclear Theory (Benjaming, Reading, MA, 1964).

[31] N. Sandulescu, N. Van Giai, and R.J. Liotta, Phys. Rev. C 61, 061301(R) (2000).

[32] J. Raynal, M.A. Melkanoff, and T. Sawada, Nucl. Phys. A101, 369 (1967).

[33] L. Ferreira, E. Maglione, and R.J. Liotta, Phys. Rev. Lett. 78, 1640 (1997).

[34] M.W. Herzog et al., Nucl. Phys. A448, 441 (1986). 